Revue internationale P.M.E.

Économie et gestion de la petite et moyenne entreprise

\title{
Le capital à risque et les jeunes entreprises innovantes : problématique et enjeux
}

\section{Bernard Guilhon et Sandra Montchaud}

Volume 16, numéro 3-4, 2003

URI : https://id.erudit.org/iderudit/1008445ar

DOI : https://doi.org/10.7202/1008445ar

Aller au sommaire du numéro

Éditeur(s)

Presses de l'Université du Québec

ISSN

0776-5436 (imprimé)

1918-9699 (numérique)

Découvrir la revue

Citer cet article

Guilhon, B. \& Montchaud, S. (2003). Le capital à risque et les jeunes entreprises innovantes : problématique et enjeux. Revue internationale P.M.E., 16(3-4),

53-73. https://doi.org/10.7202/1008445ar
Résumé de l'article

Le but de cet article est d'analyser la contribution du capital à risque à l'éclosion d'entreprises innovantes. Comme structure complexe d'intermédiation, il a pour effet de déplacer la frontière de l'innovation. Pour cela, il requiert une implication accrue de la part des investisseurs dans les étapes du choix, de la conception des contrats, et du contrôle et du suivi des participations. Au total, le capital à risque accroît l'efficience allocative dans le processus de R-D et d'innovation. 


\title{
Le capital à risque et les jeunes entreprises innovantes: problématique et enjeux
}

\author{
Bernard GUILHON \\ Sandra MONTCHAUD \\ CEFI UMR-CNRS 6126, \\ Université de la Méditerranée
}

\section{MOTS CLÉS}

\section{Capital à risque - Innovation - Asymétries d'information Compétences - Actifs incorporels - Efficience allocative}

\begin{abstract}
RÉSUMÉ
Le but de cet article est d'analyser la contribution du capital à risque à l'éclosion d'entreprises innovantes. Comme structure complexe d'intermédiation, il a pour effet de déplacer la frontière de l'innovation. Pour cela, il requiert une implication accrue de la part des investisseurs dans les étapes du choix, de la conception des contrats, et du contrôle et du suivi des participations. Au total, le capital à risque accroît l'efficience allocative dans le processus de $R-D$ et d'innovation.
\end{abstract}

\section{LES AUTEURS}

BERNARD GUILHON est professeur des Universités et responsable du DEA Économie internationale et industrielle et de la formation doctorale du CEFI, Centre d'économie et de finances internationales. II dirige le programme de recherche intitulé «Innovation et performances ». Adresse: CEFI, Château La Farge, route des Milles, 13290 Les Milles, France. Courriel : <guilhon@univ-aix.fr>.

SandRa Montchaud est attachée temporaire d'enseignement et de recherche. Ses activités de recherche concernent le capital à risque et le financement des jeunes entreprises, notamment dans les secteurs à haute intensité technologique. Adresse : CEFI, Château La Farge, route des Milles, 13290 Les Milles, France. Courriel : <sandra.montchaud@caramail.com>. 


\section{ABSTRACT}

The aim of this paper is to analyze the venture capital contribution to the start-ups creation. As a complex structure of intermediation, venture capital brings about the shifting of the innovation frontier. For this, it is required from venture capitalists to be highly involved in the screening, the design of contracts and the monitoring/ advising fonctions. All in all, venture capital upgrades allocative efficiency within the $R-D$ and innovation process.

\section{RESUMEN}

El objetivo de este papel es analizar la contribución del capital a riesgo a la eclosión de empresas innovadoras. Como estructura compleja de la mediación, causa el cambio de la frontera de la innovación. Por tanto, se requiere una implicación incrementada de parte de estos inversionistas en la selección, la elaboración de contratos, y las funciónes de control/consejo. Total, el capital a riesgo aumenta la eficiencia allocative dentro del proceso de la $R-D$ y de la innovación.

\section{ZUSAMMENFASSUNG}

Das Ziel dieses Artikels ist, den Beitrag des Risikokapitals zur Entfaltung von innovativen Unternehmungen zu analysieren. Als komplexe Vermittlerstruktur bewirkt das Risikokapital, die Innovationsgrenzen zu verschieben. Dies erfordert einen verstärkten Einfluss der Investoren in den Etappen der Wahl, der Konzeption der Verträge, der Kontrolle und der Beobachtung der Unternehmen. Insgesamt steigert das Risikokapital die effiziente Zuteilung der Ressourcen innerhalb des Forschungs- und Entwicklungsprozesse und der Innovation.

\section{Introduction}

La définition du capital à risque met l'accent sur l'idée que des ressources financières sont rassemblées par des fonds, professionnellement gérés, qui investissent dans de nouvelles entreprises pour une période de temps limitée (Amable, Paillard et Petit, 1999; Gompers et Lerner, 2001). À la dimension financière, on doit ajouter la qualité managériale : le capital à risque représente des compétences spécifiques capables de suivre et de contrôler efficacement les compétences entrepreneuriales qui explorent de nouvelles trajectoires technologiques : les technologies de l'information et de la communication, la santé et les sciences du vivant, les matériaux. (Commission européenne, 1997). Les moyens traditionnels de financement (marchés financiers et prêts bancaires) manifestent leurs limites aux États-Unis dès les années 1970, devant l'émergence de ces nouvelles technologies dont le caractère à la fois risqué et incertain exige l'intervention d'experts professionnels.

Sur le plan analytique, le capital à risque représente une structure d'intermédiation à deux étages (figure 1) : 
FIGURE 1

La structure d'intermédiation du capital à risque

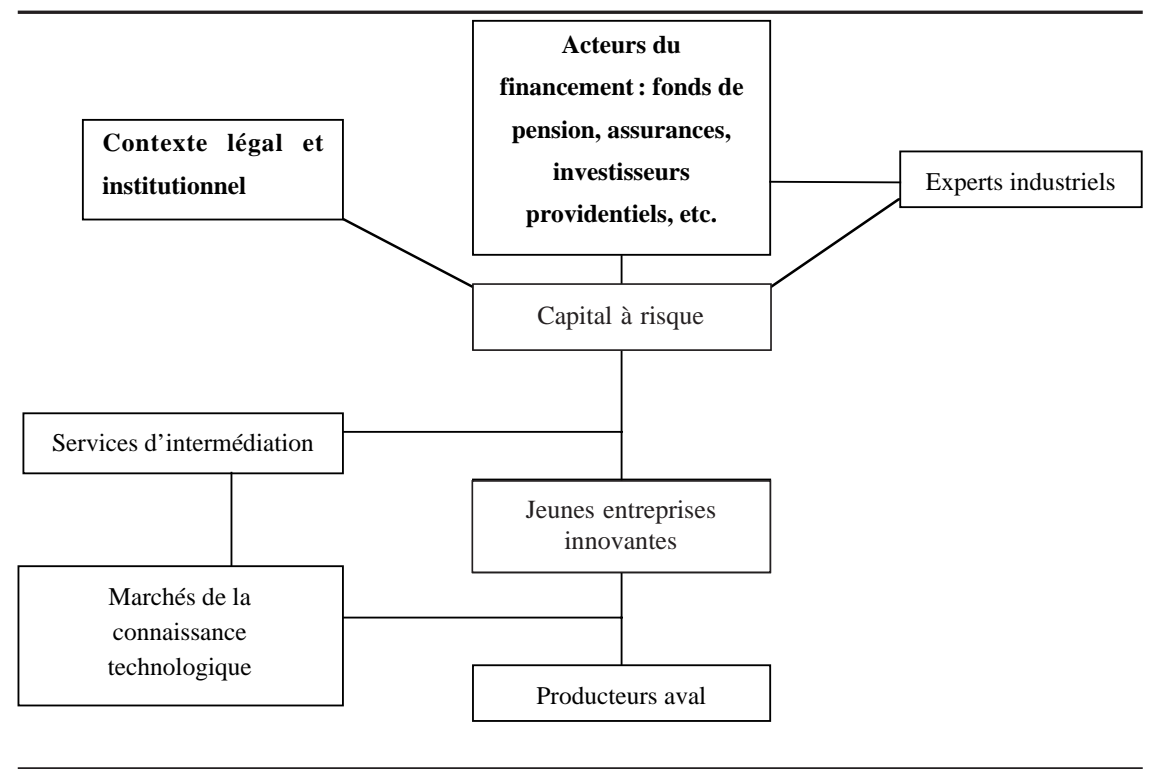

- un fonds de capital à risque est d'abord une structure de gestion de projets, fortement enracinée dans un contexte juridique et institutionnel exprimant les incitations et les limites définies par les pouvoirs publics (fiscalité, règles juridiques, mécanismes de contrôle, etc.). Sur cette base interviennent les acteurs de financement constitués essentiellement en Europe par les banques et aux États-Unis par les fonds de pension, les compagnies d'assurances, etc., auxquels il faut ajouter les investisseurs providentiels ${ }^{1}$. Le premier niveau est complété par les experts industriels dont l'intervention est souvent requise pour évaluer les perspectives de marché des projets présentés ;

- le second niveau d'intermédiation concerne plus particulièrement les projets à haute densité technologique. Depuis quelques années, on assiste à l'émergence d'institutions spécialisées dans l'intermédiation technologique qui

1. Les investisseurs providentiels représentent le capital à risque informel. Ils assurent le financement des premières dépenses en complétant les apports personnels, familiaux ou d'amis (capital à risque de proximité ou épargne de proximité) et les aides publiques obtenues avant le recours à un fonds de capital à risque. Ces investisseurs providentiels sont constitués d'anciens dirigeants d'entreprise ou de cadres supérieurs qui interviennent en amont du capital à risque (Lerner, 1998). 
jouent le rôle d'agents d'interface entre le capital à risque et les nouvelles pousses technologiques. Nombre de ces intermédiaires ont pris la forme, notamment aux États-Unis, de firmes Internet (p. ex., Venture Capital Online) qui fournissent des informations sur la qualité des projets technologiques et les possibilités de croissance. De plus, de nombreuses entreprises technologiques en démarrage, financées au départ sur une base individuelle, sont des producteurs de connaissances qui recherchent des financements complémentaires auprès des fonds de capital à risque et des informations ciblées sur les débouchés en aval (vente de licences). Dans cette perspective, l'intermédiation technologique accompagne le développement des marchés de la connaissance technologique dans de nombreuses activités: logiciels, semi-conducteurs, etc. (Guilhon et al., 2001).

Dans ce processus global, les interventions des différents acteurs peuvent avoir lieu successivement ou simultanément. Par ailleurs, la spécialisation des fonctions s'accroît au sein de ce processus, ce qui a pour effet de renforcer l'efficacité d'ensemble du dispositif, y compris dans les activités d'exploration dans lesquelles les entreprises nouvellement créées connaissent inévitablement des échecs.

Sur le plan statistique, le capital à risque peut être évalué de trois façons suivant que l'on considère les dimensions macroéconomique, sectorielle (secteurs à haute intensité technologique $v s$ secteurs traditionnels) ou les stades de développement. Le capital à risque est un sous-ensemble du capital investissement ${ }^{2}$ qui intervient tout au long du cycle de financement global de l'entreprise : l'amorçage, le démarrage (création et postcréation), le développement (l'entreprise atteint son seuil de rentabilité et dégage des profits), les transmissions / successions (rachat de l'entreprise par la direction, par les investisseurs ou par les salariés). Au sens strict, le capital à risque ${ }^{3}$ recouvre les interventions relatives aux deux premiers

2. Pour l'AFIC (2001), «est considéré comme opérateur en capital investissement tout organisme qui effectue des investissements en fonds propres dans des entreprises non cotées, s'étant doté d'une équipe de professionnels se consacrant à temps plein à cette activité et qui apporte une contribution constructive aux entreprises dans lesquelles il investit».

3. Le capital à risque «correspond aux investissements en fonds propres dans des entreprises au cours des toutes premières années de leur existence. Les financements ainsi apportés permettent de constituer la société, de financer le développement d'un premier produit ou encore de financer sa fabrication et sa commercialisation. À ce stade, l'entreprise ne génère le plus souvent aucun profit » (Les notes bleues de Bercy, 2001). 
stades : amorçage ${ }^{4}$ et démarrage, en fournissant des financements principalement pour le plan d'affaires initial, les activités de recherche, le développement du produit et la première commercialisation (European Commission, 2001).

De nombreuses études soulignent qu'au cours des dernières années l'Europe semble avoir comblé le retard qu'elle accusait par rapport aux États-Unis en termes de volume global investi dans le capital à risque (Muldur, 2001 ; European Commission, 2001). Mais la faiblesse majeure de l'Europe se situe au niveau de la répartition sectorielle des fonds investis (tableau 1). Les capitaux européens s'orientent davantage vers les secteurs à faible intensité technologique (énergie, transport, industrie, etc.), alors que la majeure partie du capital investi aux États-Unis s'oriente vers la santé / médecine et les technologies de l'information.

TABLEAU 1

Répartition sectorielle des fonds investis (\%)

\begin{tabular}{lcccc}
\hline & \multicolumn{2}{c}{ Europe } & \multicolumn{2}{c}{ États-Unis } \\
& $\mathbf{1 9 9 9}$ & $\mathbf{2 0 0 0}$ & $\mathbf{1 9 9 9}$ & $\mathbf{2 0 0 0}$ \\
\hline Santé / Médecine & 6,6 & 10,8 & 9,3 & 8,9 \\
$\begin{array}{l}\text { Technologies } \\
\text { de l'information }\end{array}$ & 24,5 & 31 & 54,7 & 60,1 \\
Autres activités & 68,9 & 58,2 & 36 & 31 \\
\hline Total & 100 & 100 & 100 & 100 \\
\hline
\end{tabular}

Source : EVCA Yearbook, 2001 et Venture Capital \& Private Equity Yearbook, 2001 / 2002.

Si l'on évalue les montants investis selon les stades de développement, on observe que l'intensité moyenne dans l'amorçage et le démarrage des projets est beaucoup plus faible en Europe qu'aux États-Unis (tableau 2).

Le but de cet article est d'analyser le capital à risque comme levier de croissance pour les jeunes entreprises innovantes. Comme mécanisme de financement, le capital à risque permet la prise en charge de projets innovants (section 1). Étant de plus en plus orienté vers des projets de ce type, il requiert une implication

4. La phase d'amorçage, souvent qualifiée de seed capital, est souvent prise en charge par les investisseurs providentiels et peut bénéficier d'aides publiques. L'amorçage représente les moyens financiers requis «pour développer une idée jusqu'au point où elle peut être techniquement et commercialement faisable » (Lachmann, 1992). Aux ÉtatsUnis, le capital à risque dans les étapes d'amorçage et de démarrage englobe le financement assuré par les investisseurs individuels et les associations créées à l'initiative des industriels (first stage financing). 


\section{TABLEAU 2}

Investissement en capital à risque dans les phases d'amorçage et de démarrage par rapport au PIB en 1999 (pour mille)

\begin{tabular}{lc}
\hline États-Unis & 1,16 \\
\hline Suède & 10,8 \\
\hline Pays-Bas & 0,91 \\
\hline Belgique & 0,90 \\
\hline Finlande & 0,56 \\
\hline Allemagne & 0,50 \\
\hline France & 0,39 \\
\hline Europe (14 pays) & 0,38 \\
\hline Royaume-Uni & 0,19 \\
\hline
\end{tabular}

Source : Commission européenne, 2001.

poussée des investisseurs dans les premières étapes de la vie d'une entreprise (section 2). L'efficience de l'allocation du capital au sein du processus de R-D et d'innovation est, de ce fait, accrue (section 3).

\section{Le déplacement de la frontière d'innovation : la nécessité du capital à risque}

La mise en œuvre de projets innovants confronte les décideurs à des situations risquées ou incertaines. Nous nous référons ici à la distinction introduite par Knight (1921) entre deux types d'incertitude : une incertitude mesurable qualifiée de risque et une incertitude non mesurable appelée incertain. Dans cette optique, pour un projet risqué, il sera possible d'affecter des probabilités d'occurrence aux divers états possibles de la nature, alors que pour un projet incertain, l'avenir n'est pas probabilisable ni de façon objective ni subjective (Herbin et al., 1998). Le problème du financement est particulièrement visible pour les entreprises innovantes comme en témoignent les résultats de l'enquête communautaire CIS 2 qui soulignent l'absence de source appropriée de financement comme cause importante du retard, de l'abandon ou du non-démarrage d'un projet innovant (Le 4 pages des statistiques industrielles, 1998 ; Bulletin de la Banque de France, 2002). Cela renvoie notamment au constat d'échec des sources de financement traditionnelles (marché ou banque) en ce qui concerne les projets innovants à forte incertitude. 


\subsection{Innovation et incertitude : les difficultés de financement}

Delaplace (1999) note qu'il serait vain de chercher un type de système financier qui favoriserait l'innovation en général et propose de mettre en parallèle l'hétérogénéité des systèmes financiers et celle des innovations. Dans cette optique, nous adopterons la distinction entre les innovations radicales et les innovations incrémentales (Freeman et Perez, 1988) ${ }^{5}$. Les innovations radicales constituent des discontinuités, des ruptures technologiques s'appuyant en principe sur des avancés scientifiques : santé / médecine (biotechnologie, imagerie médicale, etc.), technologie de l'information (matériels, microprocesseurs, logiciels, etc.), alors que les innovations incrémentales renvoient à l'amélioration de produits ou procédés. Si l'on fait l'hypothèse d'une relation positive entre l'intensité de l'innovation et le degré d'incertitude qui lui est associée notamment par les partenaires financiers potentiels, nous obtenons le graphique suivant:

FIGURE 2

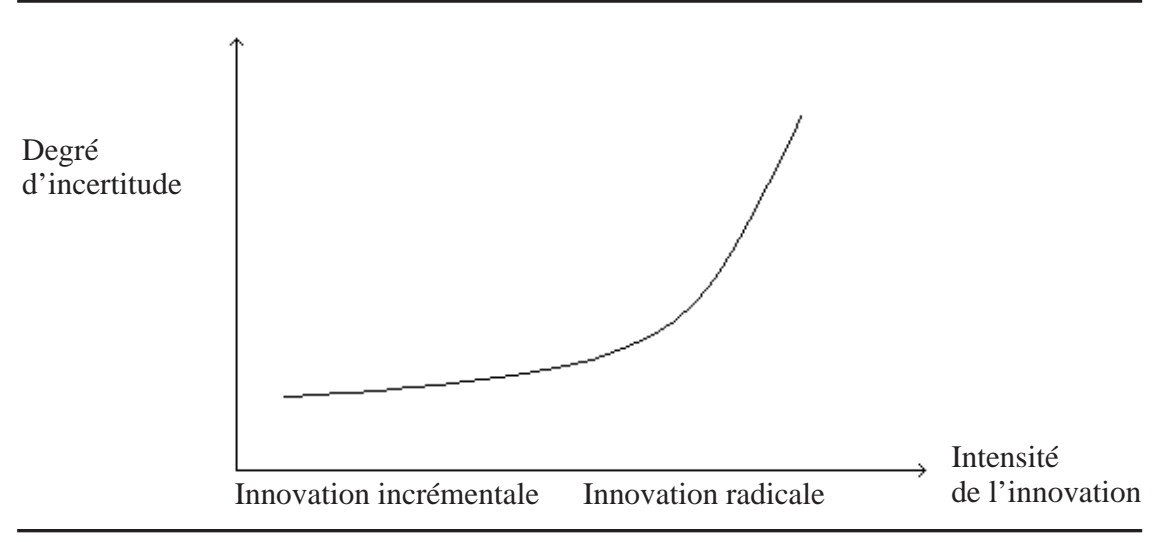

L'incertitude à laquelle nous faisons référence est à relier, d'une part, à des problèmes internes à l'entreprise, qu'ils soient de nature technologique (mise en œuvre d'une technologie nouvelle, caractère aléatoire des résultats des dépenses de R-D, etc.) ou organisationnelle (p. ex., capacité à développer et à gérer le changement) d'autre part, à la capacité d'adoption de la nouveauté par le marché. De

5. Freeman et Perez proposent la typologie suivante : innovations incrémentales, innovations radicales, nouveau «système technologique» et changements du "paradigme techno-économique» (révolution technologique), ces derniers allant de pair avec la constitution de grappes d'innovations radicales et incrémentales. Le lecteur pourra se référer à Dosi (1982) sur la distinction entre le paradigme technologique et la trajectoire technologique.

Revue internationale P.M.E., vol. 16, nºs 3-4, 2003 
façon schématique, nous pourrions donc qualifier d'incertains les projets visant à mettre en œuvre des innovations radicales et de risqués ceux qui reposent sur des innovations incrémentales. Le degré d'incertitude ainsi défini affecte à son tour les possibilités pour les entreprises d'obtenir un financement, d'où la représentation graphique suivante :

FIGURE 3

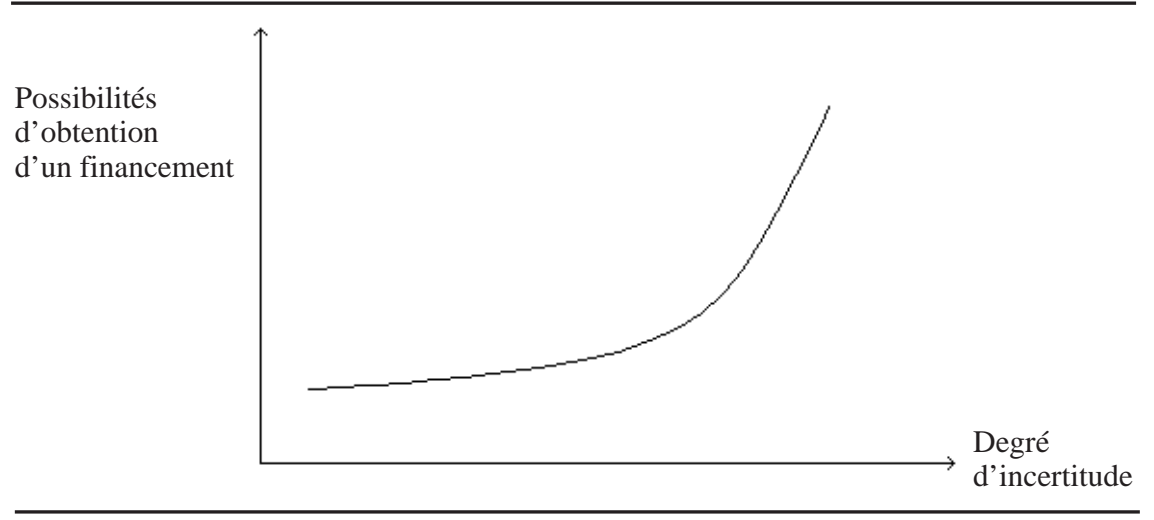

Nous pouvons résumer ce raisonnement à l'aide de la séquence suivante :

Intensité de l'innovation $\Rightarrow$ Degré d'incertitude $\Rightarrow$ Possibilités d'obtention d'un financement.

Si l'on introduit une variable supplémentaire relative à l'entreprise réclamant un financement, à savoir si le projet innovant est mis en œuvre par une firme nouvelle ou une firme établie, nous obtenons la représentation suivante :

FiguRES $4 \mathrm{a}$ et $4 \mathrm{~b}$

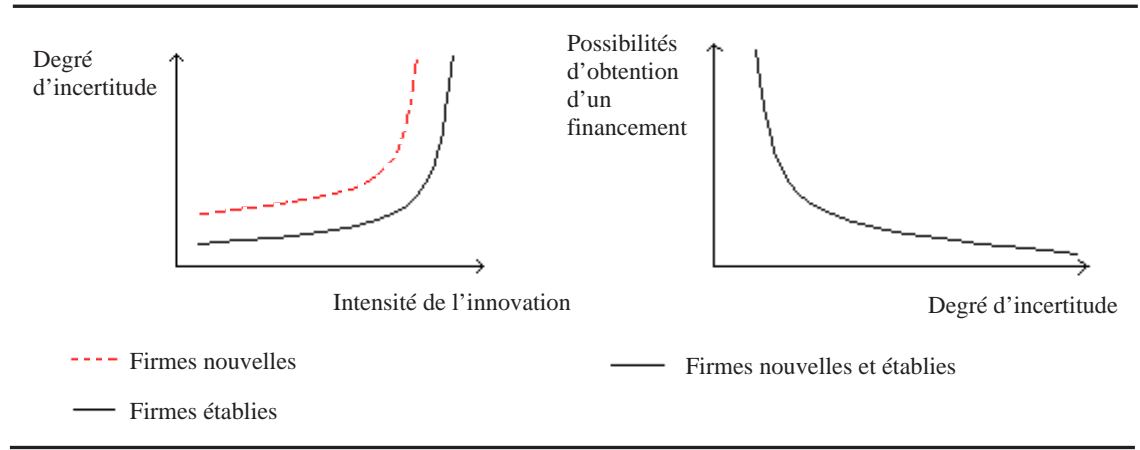

Revue internationale P.M.E., vol. 16, nos 3-4, 2003 
Pour toutes les firmes innovantes, la possibilité d'obtenir un financement est liée (négativement) au degré d'incertitude du projet perçu par l'environnement financier (figure 4b). La figure $4 \mathrm{a}$ indique que ce degré d'incertitude est déterminé par l'intensité de l'innovation, la relation entre ces deux variables étant différenciée selon l'âge des firmes. Donc, pour une intensité de l'innovation identique, le degré d'incertitude estimé serait plus grand si le projet innovant est porté par une entreprise nouvelle. De ce fait, les jeunes entreprises auraient plus de difficultés à assurer leur financement. On retrouve la proposition selon laquelle le capital à risque est le mécanisme de financement approprié pour les entreprises nouvelles de petite taille (Hall, 2002).

\subsection{La défaillance des modes de financement traditionnels}

Ces réflexions posent directement la question de l'incapacité des deux sources traditionnelles de financement d'une économie, à savoir le marché et le système bancaire, en matière d'octroi de fonds à ce type d'entreprises.

En premier lieu, le système bancaire apparaît totalement inadapté en ce qui concerne le financement des entreprises innovantes en démarrage, et cela d'autant plus si le projet de ces dernières correspond à des innovations radicales. Il est a priori plus enclin à octroyer des prêts à des firmes établies souhaitant mettre en œuvre, par exemple, une innovation incrémentale. Le rejet des jeunes pousses technologiques par ce canal de financement intermédié peut s'expliquer par les caractéristiques mêmes de ces entreprises.

Tout d'abord, les PME technologiques ne répondent pas aux critères traditionnels requis par les banques en termes d'actifs corporels (OCDE, 1986). En effet, les actifs incorporels (la R-D, les compétences techniques, les savoir-faire ou encore les idées) y sont prépondérants et ils ne constituent pas des garanties suffisantes en cas de pertes éventuelles aux yeux des prêteurs. À cela s'associe leur faiblesse en matière de fonds propres qui semble leur contraindre directement l'accès au financement bancaire (Baudry et Allegret, 1996). La logique des banquiers et celle de ces entreprises innovantes en manque de financement apparaissent être, en effet, totalement désajustées. Alors que les banquiers accordent un prêt en référence à des bénéfices passés, les entreprises en démarrage ne peuvent communiquer que leurs bénéfices potentiels futurs et elles ont souvent besoin de couvrir un déficit initial dû à de fortes dépenses de R-D.

Ensuite, et de façon complémentaire, la réticence des banques peut également s'interpréter en termes de spécificité des actifs qui tient un rôle central dans la théorie de la firme de Williamson (1988). Le fait d'innover impliquant nécessairement des actifs spécifiques pose le problème de la revente en cas de liquidation, d'où la difficulté des PME innovantes pour obtenir un prêt bancaire. Belze et 
Gauthier (2000), en référence à la théorie des coûts de transaction, rappellent que l'exigence du prêteur s'accroît dans la même mesure que le degré de spécificité de l'actif. Dans ce contexte, le recours à des contrats de dette sera plus aisé si l'on dispose d'actifs plus normalisés, c'est-à-dire moins spécifiques. De ce point de vue, le financement d'une entreprise semble refléter sa structure de production et la nature de sa politique d'investissement.

Enfin, l'existence d'asymétries informationnelles entre le partenaire financier (le principal) et l'entrepreneur (l'agent) semble jouer un rôle non négligeable au niveau de la structure de financement des entreprises. Cela signifie que les entrepreneurs disposent d'une information privilégiée sur la situation et les perspectives de développement de leur entreprise par rapport à leurs financiers. Nous distinguons deux types d'asymétries d'information:

- ex ante, c'est-à-dire avant la signature du contrat, se pose la question de la discrimination efficiente parmi les différentes demandes de financement, d'où le phénomène de sélection adverse ou d'antisélection. Stiglitz et Weiss (1981) illustrent ce problème en référence au marché du crédit sur lequel le taux d'intérêt ne constituerait pas un bon mécanisme de régulation. En effet, une hausse de ce dernier ne ferait qu'accroître le risque pour la banque en décourageant les entrepreneurs les plus sûrs (les bons risques) ou en les incitant à développer des projets plus risqués. Les banquiers sont particulièrement mal armés pour résoudre ce problème d'antisélection du fait de leurs lacunes en matière d'expertise technologique et de l'absence de réputation des jeunes entreprises, d'où des asymétries informationnelles accrues ;

- ex post, des conflits d'intérêt peuvent apparaître que l'on qualifie d'aléa moral ou risque moral. Ce problème se manifeste quand, dans le cadre d'un contrat, l'une des deux parties est en mesure de profiter de l'asymétrie d'information existante en sa faveur. L'entrepreneur peut, par exemple, mettre en œuvre un projet plus risqué que celui sur la base duquel il a obtenu son financement. L'existence de ce type de risque amplifie la frilosité des banques à l'égard des projets dont elles ne maîtrisent absolument pas les tenants et les aboutissants.

C'est donc une combinaison des différentes caractéristiques des jeunes entreprises innovantes positionnées tout particulièrement sur des secteurs à haute densité technologique (asymétrie informationnelle, intangibilité et spécificité des actifs) qui les met en marge de tout financement bancaire du fait des risques encourus et du manque de garanties matérielles.

Le tableau 3 résume les différences essentielles entre le financement par capital à risque et les prêts bancaires. 


\section{TABLEAU 3}

Système bancaire et capital à risque

\section{Prêts bancaires}

1. Investissements fondés sur des garanties matérielles, technologies éprouvées et activités traditionnelles.

2. Placements de court/moyen terme, sans intervention de la banque dans la gestion.

3. Remboursements déterminés ex ante et planifiés de manière rigide.

4. Rémunération déterminée ex ante, contractuellement, en contrepartie d'une minimisation du risque.

\section{Investissements en capital-risque}

1. Investissements fondés sur les potentialités de gains en capital contenues dans des projets novateurs.

2. Placements de long terme, impliquant un engagement direct de l'investisseur dans la gestion.

3. Retour sur investissements déterminés ex post par cession de titres.

4. Espérance de profit élevé en contrepartie d'une prise de risque importante.

Source : Bellais et Laperche, 2000.

En ce sens, le capital à risque allège la contrainte de financement des jeunes entreprises et met en avant la «proximité culturelle» entre les fournisseurs du capital-risque et les entrepreneurs innovateurs (Allegret et Dulbecco, 1998).

En second lieu, les marchés financiers, même ceux susceptibles d'accueillir les entreprises particulièrement innovantes, ne sont pas par nature destinés à financer leur naissance et les premières étapes de leur vie, mais ils peuvent intervenir par la suite pour financer leur croissance. Ils constituent, à ce titre, un maillon important dans la chaîne de financement de ce type d'entreprises. L'Europe s'est d'ailleurs dotée récemment de marchés boursiers destinés au financement d'entreprises technologiques selon le modèle américain de la NASDAQ (National Association of Securities Dealers Automated Quotation) créée en 1971. C'est en 1996 que l'EASDAQ (European Association of Securities Dealers Automated Quotation), marché paneuropéen des valeurs de croissance, a vu le jour. En offrant des possibilités de sortie aux fournisseurs du capital-risque, assurant de ce fait la liquidité de leurs placements et la réalisation de plus-values substantielles, les marchés financiers apparaissent nécessaires au bon fonctionnement de cette activité. Nous pouvons alors parler d'indissociabilité et de complémentarité entre des systèmes fondés sur le marché et ce mode de financement en fonds propres.

Ce sont donc principalement des intermédiaires financiers du haut du bilan qui vont intervenir dans des projets à forte composante immatérielle en jouant le rôle d'interface entre des investisseurs collecteurs d'épargne longue et de jeunes entreprises innovantes. Le développement de ce mode de financement, aussi bien en Europe qu'aux États-Unis, a ainsi fortement contribué au déplacement de la frontière d'innovation. 


\section{Les formes d'implication du capital à risque : le modèle à trois phases}

Le problème des asymétries d'information renvoie à la théorie de l'agence qui trouve son origine dans un article de Jensen et Meckling (1976). Les coûts d'agence ${ }^{6}$ qui en découlent apparaissent être croissants avec les actifs moins tangibles, les options de croissance et la spécificité des actifs (Gompers, 1995). L'ampleur des contraintes financières allant de pair avec les asymétries d'information, une réduction de ces dernières allégerait les contraintes de financement qui pèsent sur les entreprises innovantes. Lerner (1998) utilise le terme d' «information gap» entre les entrepreneurs et les investisseurs, et rappelle que seuls les intermédiaires financiers spécialisés tels que les fournisseurs du capital-risque peuvent résoudre ce problème en ayant recours à différents mécanismes : étude approfondie du plan d'affaires, syndicalisation, différents tours de table, contrôle, représentation au conseil d'administration, etc. Sahlman (1990) et Gompers (1995) avaient déjà insisté sur le rôle essentiel du financement par étape et des mécanismes de surveillance en tant que dispositifs de contrôle permettant de réduire ces asymétries. Dans cette optique, trois phases dans le métier d'un fournisseur du capital-risque sont communément retenues afin de permettre d'atténuer ces asymétries d'information et leurs conséquences négatives : la phase d'évaluation et de sélection, la phase de l'établissement du contrat et, enfin, celle du contrôle, du suivi et de l'apport de conseils.

\subsection{La phase de préinvestissement}

L'étude préinvestissement consiste en la recherche d'un maximum d'informations et en l'analyse approfondie du plan d'affaires. Chaque organisme de capital-risque dispose a priori de méthodes de sélection qui lui sont propres.

Kaplan et Strömberg (2000b) ont mené une étude empirique sur cette question en se basant sur un échantillon de 10 fonds regroupant 58 investissements et 42 entreprises. L'équipe managériale et l'attractivité de l'opportunité (taille du marché, stratégie, technologie, capacité d'adoption du produit par les consommateurs et la concurrence) semblent jouer un rôle important. Ils constatent, par ailleurs, que l'une des sources importantes d'incertitude que les fournisseurs du capitalrisque relèvent est le risque lié au management. Celui-ci est présent dans plus de $60 \%$ des investissements de l'échantillon, mais il n'est pas considéré comme un

6. Jensen et Meckling énoncent trois composantes des coûts d'agence : les coûts de surveillance (principal), les coûts de dédouanement (agent) et les coûts résiduels. Les auteurs précisent que le terme «surveillance» ne représente pas seulement l'observation du comportement de l'agent, mais aussi les efforts mis en œuvre par le principal afin de le contrôler au moyen de restrictions budgétaires, de politiques d'incitation. 
risque dirimant puisqu'ils ont la possibilité de modifier l'équipe de direction. Les auteurs notent, par ailleurs, une corrélation positive entre les performances de l'entreprise financée et l'analyse initiale de l'équipe faite par l'investisseur. Ils constatent, par exemple, que les entreprises bénéficiant d'une bonne appréciation sur ce point-là avant le financement ont été plus souvent introduites en Bourse.

Le problème de sélection des projets met en jeu la qualité d'expertise des investisseurs quant à la pertinence et à la viabilité du projet soumis. Il s'agit d'en évaluer les perspectives de croissance compte tenu des risques encourus. Cela nécessite des connaissances et des compétences spécialisées dans les secteurs d'activité à haute densité technologique au sein desquels des innovations tentent de voir le jour. Notons toutefois qu'au-delà de la qualité même du projet il existe des critères spécifiques à chaque organisme qui reflètent sa politique d'investissement : la taille des investissements, la localisation de l'entreprise, le stade de développement, le secteur d'activité, etc. Par exemple, le critère sectoriel est particulièrement important pour les filiales de sociétés industrielles qui ont une double logique : l'une financière avec une contrainte de rentabilité, l'autre de nature stratégique d'un point de vue productif.

Cette première phase sert non seulement à éliminer les projets non rentables ou non compatibles avec les critères spécifiques, mais aussi à déterminer les points sur lesquels le fournisseur du capital-risque pourra intervenir à travers la conception du contrat et le contrôle postinvestissement afin de valoriser au mieux l'entreprise.

\subsection{La conception des contrats}

Au moyen du contrat, les investisseurs en capital à risque vont détailler les modalités d'allocation des fonds et les mécanismes de contrôle et d'incitation qu'ils souhaitent instaurer.

Tout d'abord, ce sont les conditions d'entrée au capital qui vont être traitées, notamment le prix, le pourcentage du capital obtenu par les financiers ou encore le type de titres utilisés. Ensuite, il s'agit de conctractualiser les modalités de contrôle du fournisseur du capital-risque : représentation au conseil d'administration, présentation régulière des résultats, financement par étapes, participation aux décisions stratégiques, etc. Ces éléments peuvent faire l'objet de clauses particulières telles qu'une clause d'information comprenant la présentation de comptes trimestriels ou mensuels, la préparation de budgets prévisionnels, etc. La rédaction d'un pacte d'actionnaires vise donc à régler les relations entre les signataires en abordant des questions aussi cruciales que la sortie ou encore les moyens de surveillance, de contrôle et d'incitation. Les mécanismes d'incitation ont pour but de motiver les managers à atteindre les objectifs fixés (par exemple, l'augmentation de leur part en capital en cas de succès).

Revue internationale P.M.E., vol. 16, nos 3-4, 2003 
Le problème essentiel que cette seconde phase doit régler est celui de la répartition des droits de décision. Comment ces droits vont-ils être alloués entre l'investisseur et l'entrepreneur? Kaplan et Strömberg (2000a, 2001) ont mené une étude empirique sur 14 firmes de capital à risque impliquées dans 213 investissements répartis sur 119 entreprises. Leurs conclusions essentielles sont les suivantes :

- les financements par capital à risque permettent de modifier la répartition des droits entre les parties contractantes : il s'agit des droits de vote, des droits de siéger au conseil d'administration, des droits de liquidation et des droits sur les flux monétaires (cash-flows);

- l'exercice de ces droits dépend de mesures observables des performances financières et non financières ;

- concrètement, si les jeunes entreprises n'ont que de faibles performances, les fournisseurs du capital-risque obtiennent la totalité des droits. À l'inverse, si les performances sont jugées très bonnes, les fournisseurs du capital-risque conservent des droits sur le cash-flow, mais ils abandonnent les autres droits;

- les fournisseurs du capital-risque ont moins d'influence dans les dernières étapes du financement. Le relais peut être pris par les marchés financiers (sous la forme d'une offre publique de vente sur les nouveaux marchés) ou par les prêts bancaires (sous la forme de prêts syndiqués) lorsque l'étape du développement est atteinte.

$\mathrm{Au}$ total, les contrats sont de plus en plus conçus pour englober un ensemble de mécanismes souples et complémentaires permettant de prendre en charge les différents événements intervenant dans la vie de l'entreprise.

\subsection{Le contrôle et le suivi des participations}

Dépendants d'investisseurs soucieux de la rentabilité des fonds investis, les fournisseurs du capital-risque se doivent de pratiquer un suivi sérieux des entreprises de leur portefeuille. Il doivent faire tout particulièrement attention à la stratégie d'enracinement (managerial entrenchment; Shleifer et Vishny, 1989) de la part des dirigeants qui correspond à leur volonté de sauvegarder leur patrimoine personnel, de préserver leur place et de tirer profit de leur situation.

Du fait de l'incomplétude des contrats, nous admettrons que tout ne peut être prévu au préalable. Stéphany (2001) note, à côté d'un contrôle contractualisé, un contrôle dit informel s'appuyant sur l'influence du fournisseur du capital-risque au sein de l'environnement financier de l'entreprise et sur sa réputation qui constitue un «capital » précieux au moment d'une introduction en Bourse et qui limite, de ce fait, les comportements opportunistes de la part des entrepreneurs. 
Gompers (1995) souligne qu'un investissement associé à un fort ratio actifs intangibles / total des actifs et à une forte intensité de R-D doit faire l'objet d'un contrôle accru. Le financement par étape permet au fournisseur du capital-risque de récolter des informations et de contrôler les progrès de l'entreprise tout en conservant la possibilité d'abandonner le projet; il est à ce titre indissociable d'un processus de contrôle. Il n'est donc pas étonnant que le nombre d'étapes de financement soit particulièrement élevé pour les entreprises Internet aux États-Unis : 2418 en 1999 et 3197 en 2000 , ce qui représente respectivement $73 \%$ et $78 \%$ de l'ensemble de ces opérations réalisées au cours de ces deux années (Euromoney Publication, 2001 / 2002).

Selon Kaplan et Strömberg (2001), les fournisseurs du capital-risque sont incités à engager des activités de contrôle et de suivi coûteuses dans le but d'accroître la valeur de l'entreprise plutôt que de minimiser les possibilités de perte. Ainsi, c'est la question de la valorisation de la firme innovante qui apparaît centrale et les fournisseurs du capital-risque assistent les fondateurs en professionnalisant l'entreprise. Il reste que le contrôle exige beaucoup de temps et d'efforts et que la difficulté est accrue parce que les événements changent de nature au cours de la vie de l'entreprise. Or, les droits de contrôle se déplacent toujours vers l'investisseur en cas d'événements défavorables (Hart, 2001). Ce qui signifie qu'en cas de difficultés le fournisseur du capital-risque se réserve la possibilité de compléter l'équipe de direction avec des managers expérimentés ou de rejoindre lui-même le conseil de direction de l'entreprise.

En somme, l'innovation technologique fondée sur la mise en œuvre et la gestion d'actifs immatériels et spécifiques nécessite des compétences managériales particulières (Bulletin de la Banque de France, 1999). En ce sens et grâce à l'expertise dont le fournisseur du capital-risque doit faire preuve au cours des différentes phases, ce métier peut être considéré comme une compétence pluridisciplinaire permettant de gérer d'autres compétences (relatives notamment au projet innovant et aux technologies auxquelles il fait appel). Cette compétence pluridisciplinaire est une combinaison de compétences financières, juridiques, commerciales, technologiques, managériales, voire psychologiques. À ce titre, le capital à risque apparaît être un « financement à valeur ajoutée » représentant un gage de sérieux, de qualité et de crédibilité pour le projet et l'entreprise et cela du fait qu'il offre non seulement des capitaux, mais aussi des services à valeur ajoutée (Cherif, 1999), accroissant ainsi l'efficacité de ce mécanisme de financement.

\section{L'efficience allocative du capital à risque}

Une forme de financement renforce l'efficience de l'allocation du capital au sein du processus de recherche et d'innovation quand elle remplit deux conditions: accroître le potentiel de R-D des firmes innovantes et faciliter l'introduction des 
innovations sur le marché en favorisant ainsi la «contestation technologique » des entreprises installées (Muldur, 2001).

\subsection{L'influence du capital à risque sur le potentiel d'innovation}

Alors que l'Union européenne souffre d'une insuffisance des investissements en R-D dans les PME, aux États-Unis, on note à la fois une augmentation des dépenses de R-D privées (+8\% par année de 1995 à 2000 selon la National Science Foundation) et une forte croissance des dépenses de R-D des nouvelles firmes « technologiques » grâce au capital à risque (Gompers et Lerner, 1998). La redistribution de l'emploi des chercheurs confirme cette tendance : en 1999, $33 \%$ des chercheurs scientifiques travaillaient dans le secteur privé dans des entreprises ayant moins de 500 salariés, alors qu'ils n'étaient que $16 \%$ en 1993.

Le développement du capital à risque transforme l'organisation du processus d'innovation. Des inefficiences importantes semblent se manifester dans les dépenses en capital et de R-D d'un nombre important de grandes entreprises (Jensen, 1993). Le processus de R-D interne, souvent organisé autour d'un laboratoire central, est censé permettre d'exploiter la complexité et la cumulativité de la connaissance technologique (Teece, 1988). Cependant, face à des ruptures technologiques, d'autres pratiques sont plus appropriées pour saisir les opportunités technologiques : joint-ventures, collaborations avec les universités, essaimages, recherche contractuelle, capital à risque.

De façon générale, le capital à risque accroît l'efficience allocative sur deux plans. D'une part, à un processus de R-D interne et centralisé susceptible d'engendrer des coûts substantiels d'opportunité (beaucoup de projets n'aboutissent pas selon Gompers et Lerner, 2001) se substituent des «investisseurs actifs » et sélectifs ayant une connaissance fine des projets et des capacités d'expertise (Jensen, 1993). D’autre part, grâce aux dépenses de R-D réalisées par les jeunes entreprises, une division du travail innovatif se met en place dans de nombreuses activités (biotechnologies, technologies de l'information et de la communication, etc.). Les petites entreprises «explorent» les trajectoires technologiques nouvelles alors que les grandes entreprises installées «exploitent» les trajectoires technologiques existantes (Arora, Fosfuri et Gambardella, 2001). Le phénomène est particulièrement marqué dans les technologies de l'information où les jeunes entreprises sont une source essentielle de création de nouveautés. L'orientation de la majeure partie du capital à risque vers les entreprises de logiciels et de services, plus spécialement vers les entreprises Internet (tableau 4), renforce donc le potentiel d'innovation de l'économie américaine. 
TABLEAU 4

Investissements en capital à risque aux États-Unis (en millions de dollars)

\begin{tabular}{lcc}
\hline & $\begin{array}{c}\mathbf{1 9 9 6} \\
\mathbf{1}^{\text {er }} \text { semestre }\end{array}$ & $\begin{array}{c}\mathbf{2 0 0 0} \\
\mathbf{1}^{\text {er }} \text { semestre }\end{array}$ \\
\hline Investissements totaux & 4143 & 36908 \\
Investissements dans les entreprises Internet & 413 & 22704 \\
\hline
\end{tabular}

Source : Euromoney Publication, $2001 / 2002$.

La croissance est spectaculaire : en 1996, les investissements dans les entreprises Internet représentaient moins de $10 \%$ de l'investissement total et $61,5 \%$ de ce même total en 2000. Grâce à ce mécanisme, un double processus de spécialisation se met en place. Dans l'industrie du logiciel, les produits sont conçus et réalisés soit par des fabricants de systèmes informatiques qui vendent à la fois des matériels et des logiciels (IBM, Sun microsystems, etc., ce que nous appelons spécialisation relative) ou par des fabricants de logiciels indépendants (Independent Software Vendors ou ISV) qui produisent exclusivement des logiciels (spécialisation absolue : PeopleSoft, Oracle, etc.). Par ailleurs, il est intéressant de remarquer que le revenu dépensé en R-D est proportionnellement moins important dans le cas des grandes firmes (US Industry and Trade Outlook, 2000). Tout se passe comme si le capital à risque avait pour effet de redistribuer les coûts et les risques du financement de la R-D entre les entreprises, en fournissant des fonds à des jeunes entreprises en forte croissance qui ont des plans d'activité viables et des perspectives de marché élevées.

\subsection{L’introduction des innovations sur le marché}

L'industrie du capital à risque, malgré des débuts difficiles (faibles performances atteintes par le programme SBIC, Small Business Investment Company, durant les années 1980 et le début des années 1970), a pris son essor grâce aux montants investis à la fin des années 1970 et au début des années 1980 (Gompers et Lerner, 2001) et aux politiques publiques mises en œuvre (le progamme SBIR, Small Business Innovation Research, création de 107 incubateurs dans une quinzaine d'États). De nouvelles entreprises se créent qui repèrent des niches de marché et entrent ainsi dans certaines activités par innovation. Ces entrepreneurs schumpétériens s'appuient sur les circuits du financement à risque soutenus soit par le gouvernement (Apple, Intel, Federal Express, Compaq, etc.), soit par le secteur financier privé (Microsoft, Digital Equipment, Computer Vision, etc.). Ces firmes vont connaître une croissance rapide et vont favoriser le renouvellement de la base technologique de l'industrie américaine. La structure industrielle présente la

Revue internationale P.M.E., vol. 16, nos 3-4, 2003 
particularité, par rapport aux pays de l'Union européenne, d'être constituée d'un nombre important de PME disposant de capacités de R-D et d'innovation et explorant un large éventail de trajectoires technologiques.

$\mathrm{Au}$ sein de cette dynamique, on observe un double phénomène. D'une part, le temps mis par un produit pour atteindre le marché est sensiblement réduit dans les entreprises financées par du capital à risque (Hellman et Puri, 2000). D'autre part, une redistribution des cartes s'opère au détriment des entreprises en place : ces dernières sont l'objet d'une «contestation technologique » par des entreprises nouvelles, souvent de taille modeste, capables de fournir « un ensemble d'innovations complémentaires » (logiciels, microprocesseurs, systèmes de communications, etc. ; Baily et Lawrence, 2001). Outre l'effet de renouvellement de la structure industrielle, il en résulte un avantage compétitif global dans les industries émergentes caractérisées par une forte concurrence et des configurations technologiques non encore stabilisées.

L'impact du capital à risque sur l'efficience allocative peut être estimée d'une autre façon. Kortum et Lerner (1998), utilisant à la fois des données par firme et par industrie, observent que l'activité de brevet est stimulée davantage par le capital à risque que par la R-D des grandes entreprises. Par ailleurs, les estimations économétriques de l'impact d'un dollar de capital à risque par rapport à un dollar de R-D démontrent que le capital à risque représente $15 \%$ de l'innovation industrielle aux États-Unis dans la décennie précédente. Les comportements d'innovation sont donc bien différenciés entre les entreprises adossées à du capital à risque et les autres.

\section{Conclusion}

Le capital à risque s'est progressivement imposé comme une forme appropriée de financement pour les entreprises innovantes en supplantant les modes traditionnels de financement (marchés financiers et prêts bancaires) et en apparaissant comme un mécanisme original combinant les forces des deux systèmes, le marché et la banque (Hall, 2002). D'une part, le capital à risque fournit des stimulants puissants qui placent l'entrepreneur dans un contexte de marché. D'autre part, la surveillance exercée par un investisseur informé apparaît comme la caractéristique d'un système fondé sur la banque. En effet, l'éclosion des jeunes entreprises présente des difficultés particulières dans le cas de projets fortement innovants. La rentabilité extrêmement incertaine des investissements et le fait que les décisions ne puissent pas être fondées sur des résultats passés mais sur des perspectives de croissance font du capital à risque une structure d'intermédiation au sein de laquelle les jugements des experts et des professionnels conditionnent fortement les possibilités d'engagement. 
Ces investisseurs s'impliquent de façon importante dans la création et le démarrage des jeunes entreprises. La forte relation bilatérale construite entre un fournisseur du capital-risque et un entrepreneur a pour effet de réduire les asymétries d'information dues notamment à la présence massive d'actifs incorporels. Elle requiert également une sélectivité accrue et l'introduction de mécanismes complémentaires permettant de faire face à des situations défavorables. Elle est aussi synonyme d'offre de services managériaux concernant plus particulièrement le contrôle et le suivi dans les premières années de la vie de l'entreprise. L'efficacité des investissements en capital à risque peut ainsi être augmentée.

Malgré cette efficacité accrue et l'existence de relations fortes entre l'investisseur et l'entrepreneur, il reste que de jeunes entreprises échouent au bout de quelques années parce que le risque de défaillance a été largement sous-estimé. En surestimant la qualité technologique des projets qui se développent au sein de la vague d'innovations centrées sur les technologies de l'information et en privilégiant exagérément la notion de débouché, il se peut que des moyens de financement aient profité à des entreprises non rentables. Si cet effet d'éviction par rapport aux secteurs traditionnels était confirmé, il tendrait à relativiser l'argumentation concernant l'efficience de l'allocation du capital.

\section{Bibliographie}

AFIC (2001), Rapport sur l'activité du capital investissement en France 2000.

Allegret, J.-P. et P. DubelCCO (1998), «Le comportement de la firme innovante: structure de gouvernance et mode de financement », Revue d'économie industrielle, $\mathrm{n}^{\circ} 84$, p. $7-25$.

Amable, B., S. PAillard et P. Petit (1999), « Finance and innovation : venturing on the risk frontier», Rapport dans le cadre d'un programme T.S.E.R., Communauté européenne, D.G.XII, Bruxelles.

Arora, A., A. Fosfuri et A. GAMBARdella (2001), Markets for Technology, Cambridge, Mass., The MIT Press.

BAILY, M.B. et R.Z. LAWRENCE (2001), « Do we have a new e-conomy ? , NBER Working Paper $n^{\circ} 8243$, Cambridge.

BAUDRY, B. et J.-P. Allegret (1996), «La relation banque-entreprise: structures de gouvernement et formes de coordination », Revue française d'économie, vol. 11, $\mathrm{n}^{\mathrm{o}} 4$, p. 3-36.

BELLAIS, R. et B. LAPERCHE (2000), «Entrepreneurs innovateurs, capital-risque et croissance des grandes entreprises », Innovations, Cahiers d'économie de l'innovation, $\mathrm{n}^{\circ} 12, \mathrm{p} .137-155$.

BELzE, L. et O. GAUTHIER (2000), «Innovation et croissance économique : rôle et enjeux du financement des P.M.E. », Revue internationale PME, vol. 13, nº 1, p 64-86.

BULLETIN DE LA BANQUE DE FRANCE (1999), Le financement de l'innovation, nº 65.

Revue internationale P.M.E., vol. 16, nos 3-4, 2003 
BUlletin DE LA BANQUE DE FRANCE (2002), Financement des entreprises industrielles innovantes: contraintes financières et risque, $\mathrm{n}^{\circ} 98$.

CHERIF, M. (1999), «Capital risque et valorisation de la firme innovante non cotée », Les Cahiers du Gratice, no 16, p. 43-71.

COMMISSION EUROPÉENNE (1997), Le Deuxième rapport sur les indicateurs scientifiques et technologiques, D.G.XII, Office des publications officielles des Communautés européennes, Bruxelles.

COMPANIES AND U.S. DEPARTMENT OF COMMERCE - INTERNATIONAL TRADE ADMINISTRATION (2000), U.S. Industry and Trade Outlook, New York, McGraw-Hill.

DElaPlaCE, M. (1999), «Hétérogénéité des innovations et hétérogénéité des systèmes financiers », Les Cahiers du Gratice, ${ }^{\circ}$ 16, p. 23-41.

DosI, G. (1982), «Technological paradigms and technological trajectories», Research Policy, vol. 2, no 3, p. 147-162.

Euromoney Publication (2001/2002), Venture Capital \& Private Equity Yearbook, D. Robinson (dir.), Londres.

European Commission (2001), Key Figures 2001, Special Edition, Indicators for Benchmarking of National Research Policies, European Communities, Research Directorate General, Brussels.

EVCA Yearbook (2001), Annual Survey of Pan-European Private Equity \& Venture Capital Activity.

Freeman, C. et C. Perez (1988), «Structural crises of adjustment, business cycles and investment behaviour », dans G. Dosi et al. (dir.), Technical Change and Economic Theory, Londres, Pinter Publishers, p. 38-66.

GOMPERS, P.A. (1995), «Optimal investment, monitoring, and the staging of venture capital», The Journal of Finance, vol. 50, no 5, p. 1461-1489.

GOMPERS, P.A. et J. LERNER (1998), «The determinant of corporate venture capital successes : organizational structure, incentives, and complementarities », NBER Working Paper $n^{\circ}$ 6725, University of Chicago.

GOMPERS, P.A. et J. LERNER (2001), «The venture capital revolution», Journal of Economic Perspectives, vol. 15, $\mathrm{n}^{\mathrm{o}}$ 2, p. 145-168.

Guilhon, B. et al. (2001), Technology and Markets for Knowledge, Boston, Kluwer Academic Publishers.

Guilhon, B. et S. Montchaud (2003), «The venture capital in the new economy», dans New Economy Handbook, Amsterdam, Academic Press, Elsevier Science.

HALl, B.H. (2002), «The financing of research and development», Oxford Review of Economic Policy, vol. 18, no 1, p. 35-51.

HART, O. (2001), «Financial contracting », Journal of Economic Literature, vol. 39, nº 4, p. 1079-1100.

Hellman, T. et M. PURI (2000), «The interaction between product market and financial strategy. The role of venture capital», Review of Financial Studies, vol. 2, p. $959-984$.

Revue internationale P.M.E., vol. 16, $\mathrm{n}^{\text {os }}$ 3-4, 2003

(C) 2004 - Presses de l'Université du Québec

Édifice Le Delta I, 2875, boul. Laurier, bureau 450, Sainte-Foy, Québec G1V 2M2 • Tél. : (418) 657-4399 - www.puq.ca

Tiré de : Revue internationale P.M.E., vol. 16, nos 3-4, sous la direction de Pierre-André Julien • PME1603N Tous droits de reproduction, de traduction et d'adaptation réservés 
Herbin, F., C. Bailleul, O. Jokung et P. N'Gahane (1998), Finance et placements, Paris, Armand Colin, 224 p.

JENSEN, M.C. (1993), «Presidential address : the modern industrial revolution, exit, and the failure of internal control systems », The Journal of Finance, vol. 48, n 3 , p. 831-880.

JENSEN, M.C. et W.H. MECKLING (1976), «Theory of the firm: managerial behavior, agency costs and ownership structure », Journal of Financial Economics, vol. 3, $\mathrm{n}^{\mathrm{o}} 4$, p. 305-360.

KAPLAN, S.N. et P. STRÖMBERG (2000a), «Financial contracting theory meets the real world : an empirical analysis of venture capital contracts », NBER Working Paper $n^{o} 7660$, University of Chicago.

KAPLAN, S.N. et P. STRÖMBERG (2000b), « How do venture capitalists choose and manage their investments?», NBER Working Paper, University of Chicago.

KAPLAN, S.N. et P. STRÖMBERG (2001), «Venture capitalists as principals : contracting, screening, and monitoring », NBER Working Paper $n^{\circ} 8202$, University of Chicago.

KNIGHT, F.H. (1921), Risk, Uncertainty, and Profit, Londres, Houghton Mifflin Company.

KORTUM, S. et J. LERNER (1998), «Does venture capital spur innovation ? , NBER Working Paper $n^{\circ}$ 6846, Cambridge.

LACHMANN, J. (1992), Le seed capital: une nouvelle forme de capital risque, Paris, Economica.

LE 4 PAGES DES STATISTIQUES INDUSTRIELLES (1998), L'innovation technologique progresse dans l'industrie, $\mathrm{n}^{\circ} 89$.

LERNER, J. (1998), « Angel financing and public policy : an overview », Journal of Banking and Finance, vol. 22, p. 773-783.

LES NOTES BLEUES DE BERCY (2001), «Le tableau de bord de l'innovation », no 219.

MULDUR, U. (2001), «Is capital optimally allocated in the overall process of European innovation?, Revue d'économie industrielle, no 94, p. 115-153.

OCDE (1986), Le capital risque, contexte, développement et action des pouvoirs publics.

SAHLMAN, W.A. (1990), « The structure and governance of venture capital organizations », Journal of Financial Economics, vol. 27, n 2, p. 473-521.

SHLEIFER, A. et R.W. VISHNY (1989), « Management entrenchment : the case of managers specific investments », Journal of Financial Economics, vol. 25, p. 123-139.

StÉPHANy, E. (2001), «L'évolution des pratiques du capital risque en France», Revue française de gestion, $\mathrm{n}^{\mathrm{o}} 135$, p. 63-75.

STIGLITZ, F. et A. WEISS (1981), «Credit rationing in markets with imperfect information », The American Economic Review, vol. 71, n 3, p. 393-410.

TEECE, D.J. (1988), « Technological change and the nature of the firm », dans G. Dosi et al. (dir.), Technical Change and Economic Theory, chapitre 12, 4e partie, Londres, Pinter Publishers.

Williamson, O.E. (1988), «Corporate finance and corporate governance », Journal of Finance, vol. 43, p. 567-591.

Revue internationale P.M.E., vol. 16, nos 3-4, 2003

(C) 2004 - Presses de l'Université du Québec

Édifice Le Delta I, 2875, boul. Laurier, bureau 450, Sainte-Foy, Québec G1V 2M2 • Tél. : (418) 657-4399 - www.puq.ca

Tiré de : Revue internationale P.M.E., vol. 16, nos 3-4, sous la direction de Pierre-André Julien • PME1603N Tous droits de reproduction, de traduction et d'adaptation réservés 